\title{
IN-FACTORY LEARNING - QUALIFICATION FOR THE FACTORY OF THE FUTURE
}

\author{
QUINT Fabian \\ Innovative Factory Systems, German Research Center for Artificial Intelligence DFKI, Kaiserslautern, \\ Germany, fabian.quint@dfki.de \\ MURA Katharina \\ Innovative Factory Systems, German Research Center for Artificial Intelligence DFKI, Kaiserslautern, \\ Germany, katharina.mura@dfki.de \\ GORECKY Dominic \\ Innovative Factory Systems, German Research Center for Artificial Intelligence DFKI, Kaiserslautern, \\ Germany, dominic.gorecky@dfki.de
}

\begin{abstract}
The Industry 4.0 vision anticipates that internet technologies will find their way into future factories replacing traditional components by dynamic and intelligent cyber-physical systems (CPS) that combine the physical objects with their digital representation. Reducing the gap between the real and digital world makes the factory environment more flexible, more adaptive, but also more complex for the human workers. Future workers require interdisciplinary competencies from engineering, information technology, and computer science in order to understand and manage the diverse interrelations between physical objects and their digital counterpart. This paper proposes a mixed-reality based learning environment, which combines physical objects and visualisation of digital content via Augmented Reality. It uses reality-based interaction in order to make the dynamic interrelations between real and digital factory visible and tangible. We argue that our learning system does not work as a stand-alone solution, but should fit into existing academic and advanced training curricula.
\end{abstract}

Key words: Training Systems, Advanced Interaction, Digital Factory, Factory of the Future, Virtual Reality, Industry 4.0

\section{Introduction}

The arising connection between the real physical world (like our homes and factories) and the digital world (support by internet technologies) brings more and more intelligent objects into our everyday lives and work contexts. The so-called Internet of Things facilitates, for instance, smart homes, which can adjust heat, light, and ventilation autonomously in order to optimize both energy consumption and comfort. The Internet of Things implies a revolution for the future factory environment, too. Following the Industry 4.0 vision [1] we expect the digitalisation to promote intelligent devices, which are able to learn from experiences, to communicate with each other, and to take decisions towards selfoptimization. Alongside this extensive automation, the human worker has been acknowledged as the most flexible entity in the production system, who plans, controls, manages, and trouble shoots. Thus, the demands for the employees' broad and interdisciplinary skills increase simultaneously.

High complexity and various interdependencies result from the involved smart objects, which may act in a dynamic way instead of being static and predictable. Furthermore, production workers will initially have difficulties to keep track of the digital information behind the physical production environment. The challenge is that the digital mechanisms are invisible in nature. Professions in manufacturing or electronic engineering have to be enriched by interdisciplinary competences from computer science in order to make employees cope with their new requirements. Therefore, innovative and appropriate qualification is needed.

In the light of the mentioned learning challenges within smart factories and other environments, we suggest a comprehensive approach combining current trends from human-machine interaction and insights from psychological and pedagogical science with the aim of developing interactive learning environments integrating real and virtual world. 


\section{Motivation}

Future production workers must be enabled to act in a world that combines real devices and digital content. For instance, usage of Cyber-physical systems (CPS) in the factory involves understanding of classical production engineering but also competences in internet, sensor, and information technologies.

Classical learning environments for engineers are rather formal with focus on acquisition of theoretical knowledge, e.g., by seminars and E-Learning tools. Transfer to the real factory environment is inhibited due to lack of practical experiences and exploration of the newly acquainted knowledge. Furthermore, E- Learning offers only limited user interaction possibilities restricted to point-and-click interactions. We argue that teaching the complex interplay between real and digital world, i.e., the principles of the Internet of Things, requires that the learning environment incorporates both worlds, too. After reviewing the current State of the Art, this paper presents a learning environment based on mixed reality, which makes experiencing the Internet of Things paradigms possible. It combines the flexible and extensive presentation of virtual information together with real world objects, which enable actual experience of physical consequences. Our approach further incorporates ideas for integrating such a learning environment into existing educational and organizational processes.

\section{State of the Art}

\section{Mixed Reality}

Mixed reality environments contain a combination of real and virtual world information. First applications in the context of production were virtual assembly instructions augmenting the real workspace in order to support assembly workers in air plane manufacturing [2]. This context-sensitive overlay of virtual objects over the real environment is known as Augmented Reality. Other mixed reality applications emphasize the virtual content without connecting it to the real environment, i.e., Virtual Reality systems. The potential of both Augmented and Virtual Reality for learning purposes has been elaborated in previous work, i.e., COGNITO [3], VISTRA [4], AR handbook [5], and manual workstation [6]. For instance, using head mounted displays or glasses providing information at the time and place when and where it is needed can be a promising opportunity for learning on-the-job. Furthermore, assembly tasks can be practiced in virtual environments [4]. Additional enhancements regarding pedagogical concepts enhance the suitability for learning [7].

However, the Industry 4.0 paradigm will not only require shop floor personnel to be trained efficiently but rather addresses all workers to understand the principles and paradigms of an arising networked, digitalized Factory of Things. A recently published concept was developed to make the characteristics of the Internet of Things tangible, i.e., users can experience the merging of real and virtual world [8]. We argue that there is high potential for such a mixed reality approach for learning and qualification purposes, i.e., making the Industry 4.0 paradigm experienceable and tangible.

\section{Trends towards reality-based interaction and gamification}

New developments in human-machine interaction stress the importance of replacing simple „point-and- click“ interactions - i.e., mouse, desktop, and keyboard used in classical E-Learning environments - by

„being-in-the-world“ and „thinking-through-doing“ approaches, i.e., the possibility to actually interact with physical and virtual objects [9]. These concepts have been noted reality-based interaction [10], blended interaction [11] or embodied interaction [9] and they address a tangible learning experience by encouraging to actually handle with and make use of objects. These bodily experiences have been shown to positively impact learning related cognitive processes. Reality-based interaction which combines physical experiences with digital information seems to be the means of choice for getting across the Internet of Things, which likewise is the combination of real and virtual world [8]. Yet, its potential for learning purposes has not been exploited. First interactive applications in the industrial field indeed integrate real und virtual world [12], [13] but they lack elaborated learning contents, pedagogical concepts, and appropriate hard- and software use.

Another important factor is motivation of users to actually interact within the learning environment. One way to address this is usage of gamification elements [14], which are playful features enabling new ways of knowledge communication, increasing experiences of being involved, motivating, and offering incentives for learning and performance. Despite many applications of such „Serious 
Games" in the context of learning [15], there is no concept for using gamification appropriately within the Industry 4.0 era [16].

\section{Blended learning}

Successful learning concepts in the industrial domain are summarized under the term blended learning. They combine formal theoretical sessions [17] with active testing and self-exploration possibilities [18], [19], instead of only teaching theory in seminars. For instance, academic curricula for engineering studies incorporate regular tutorials, in which students practically apply their knowledge acquired in lectures. In the context of further education for professionals, who already worked in production for several years, practically relevant learning content can be taught in so-called "teaching and learning factories”. They represent a realistic factory environment which encourages employees to try out different activities or decisions and experience their consequences [20]. The main goal is the development of competences, i.e., knowledge, skills, motivation, interest, abilities, and behaviors related to the requirements of the specific working tasks [21]. In order to achieve this, teaching and learning factories apply additional concepts like

„mistakes as positive learning experience“ [22] and social team learning [23]. The learning content is limited to the extent that only knowledge is taught, which is important in nowadays factories. There are no extensive learning concepts to teach the complex interplay between real and virtual world in the factory of the future.

\section{Proposed Concept}

\section{Vision}

We suggest that technologies in human-machine interaction like mixed reality concepts, realitybased interaction, and gamification are promising to be integrated into new learning environments, which address the challenges of the Factory of Things.

Our vision is an interactive, mixed reality based learning environment, which makes these complex and invisible relations experienceable and thereby understandable for todays and tomorrow's production environments. Thereby, new possibilities regarding communication and optimization or the enhancement of physical objects by software components towards smart objects can be explored, played through, and experienced. As discussed in the previous chapters, the requirements towards the worker continuously change and demand in future a broader understanding and competences-oriented learning comprising several disciplines from engineering and information technology. Therefore, the next intruding evolution is to use existing Augmented Reality technologies to represent the digital world and combine this with various real world elements. These real world elements are primarily any kind of smart objects such as CPS in the industrial domain or other embedded devices in the consumer domain.

All of these smart objects have in common that they realize a dedicated subtask in a decentralized network. For the responsible person managing such a network in a productive environment it is inevitable to have a strong and profound overall understanding of the resulting interdependencies. Otherwise, it will not be possible to take full advantage of the new paradigms and resulting benefits. Since our vision of the learning environment should not address one specific discipline but help various people from several disciplines to gain a deeper understanding we propose a modular programming framework for the CPS. Therefore, previous ideas and concepts of an app-based framework for modular programming as shown in [24] are further developed to be used as the basis of this programming framework. By using code snippets, basically meaning software components with encapsulated functionality, we put less focus on programming aspects but rather on understanding the interdependencies of these functionalities. Thus, we also incorporate beginners and other persons, who are not directly related to programming as for example an automation engineer. Furthermore, the learning content should not promote only one feasible solution, since in real world several solutions are possible where depending on the circumstances and requirements the best one has to be determined. This means that the content needs to be structured in such a way that the user is motivated to try out the different degrees of freedom, to tweak on different adjusting screws, and experience the resulting consequences.

The user is encouraged to push the system to its limits, try out risky or error-prone configurations, make faults and eventually learn from these. Approaches from gamification inspire the playful interaction with the learning content and gain additional motivation. Furthermore, the individual differences of the learner regarding demographic factors und available qualification and prior knowledge needs to be considered. Learning sessions with varying content, degree of interactivity or difficulty level enable a self-controlled adjustment to the own state of knowledge and interests in advanced training. The 
promoting elements are presented to the learner by using mixed reality in an intuitive and self-explaining manner, so that the learning content is both challenging as well as manageable.

\section{System Architecture}

The proposed system architecture, as shown in Figure 1, comprises a set of CPS, which are configured/programmed via an appropriate framework (CPS Framework). An Augmented Reality Engine (AR Engine) handles the AR content representing the digital domain. A code database (Code DB) provides the underlying software components. The CPS components can be equipped with different sorts of sensors and actuators such as proximity sensors, light barriers, servos, and many more. Our CPS components were developed within several research projects and consist of an off-the-shelf System-onModule, which is combined with a customized circuit board providing GPIOs and other connectors for attaching external devices. For running the code that realizes the functionality of each CPS a Linux-based operating system is used. The encapsulated and pre-compiled software components referred to as code snippets (mentioned in the previous section) are stored in the code database (Code DB). These single code snippets are retrieved by the CPS Framework, put together, and installed on the respective CPS based on the user's configuration. The interface to make the configuration is also given by the CPS Framework and can be provided to the user on a suited human-machine interface (HMI), here represented by a tablet. Once the configuration is done, the CPS Framework keeps track of the current overall system status and the current parameters. They are further processed within the AR Engine that provides the AR content towards the HMI. This could again be the same tablet used for configuration or any other modality providing AR capabilities such as Smart Glasses.

Figure 1: Concept of the mixed-reality learning environment

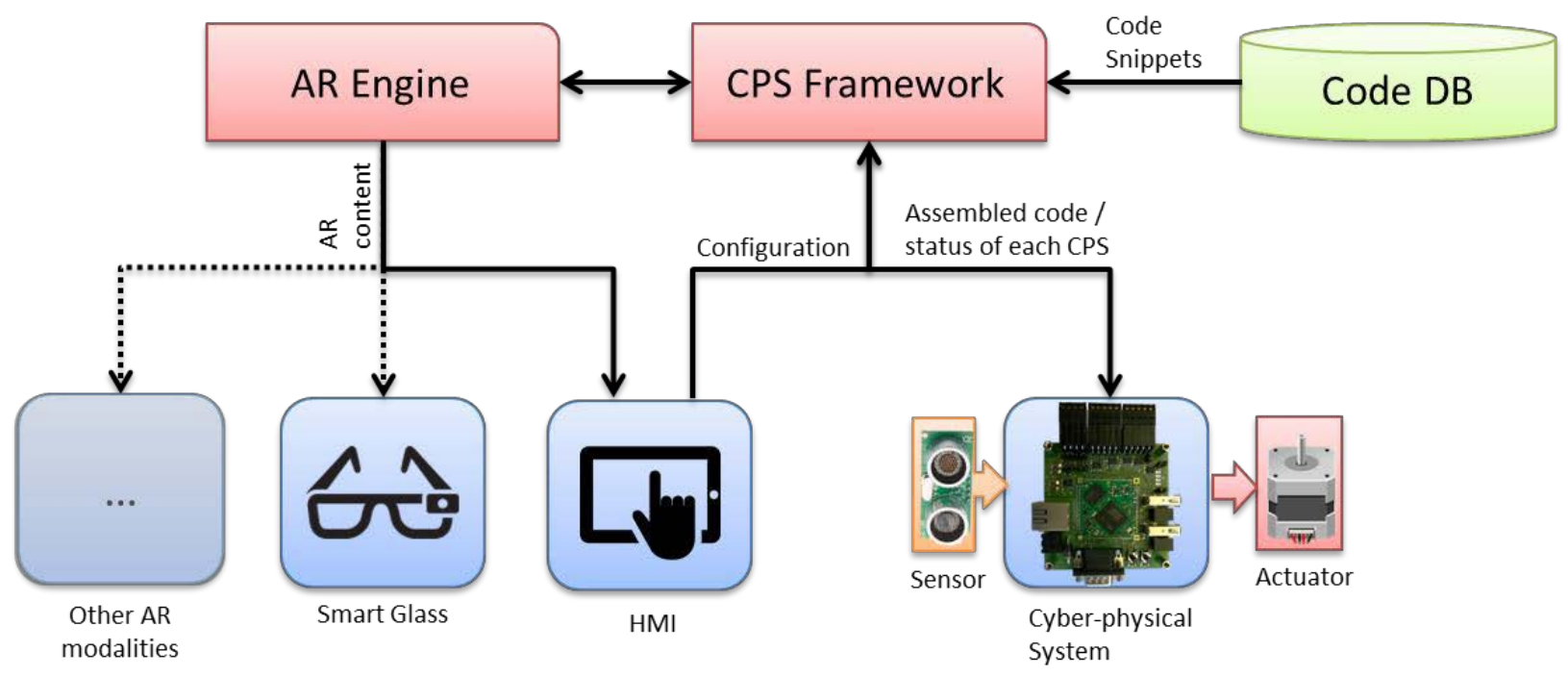

\section{Use Case}

The learner chooses one of the possible learning session by using the tablet. He wants to learn how sensors, actuators need to be connected to a CPS and how these communicate with each other by designing and setting up a simple production process composed of three steps (e.g. drilling holes, fixing screws, and a final quality check). Before starting the configuration the learner needs to work out an idea how to realize the required production process in a decentralized manner. For this purpose he can use several CPS, sensors/actuators, and code snippets. So he needs to develop a concept which CPS takes over which sub-task of the overall process, which sensors/actuators are required, and how the code snippets need to be configured accordingly. Once this is done he connects the sensor/actuators to the CPS and brings them in the designated process order. Afterwards, he starts the configuration user interface (UI) provided through the tablet, where the software programming of the CPS is done by choosing and configuring the code snippets. These code snippets are illustrated by self-explaining icons which represent the respective encapsulated functionality. So he needs to align these icons in a possible order for each of the CPS. Besides that parameters related to the depicted production can be changed from the default values (e.g. throughput, processing time). Once the configuration was finished the CPS components are fitted with the resulting software. Afterwards, the system behaviour can be experienced. All process steps are simulated by vivid animations representing the digital consequences, enhancing the real interactions made tangible by the sensors and actuators of the CPS. Thus, the user can always understand the current process step and status of the system, since direct feedback is provided at all times 
by augmenting the physical world with the digital. Furthermore, he can adjust the made configuration and come step-by-step to the best solution for the posed constraints.

\section{Summary and Outlook}

The challenges of the proceeding application of the Factory of Things require manufacturing enterprises to invest in advanced training of their employees. Since future workers require interdisciplinary competencies from multiple domains such as engineering, information technology, and computer science, these aspects have to covered by convenient learning environments. Otherwise, it will not be possible to take full advantage of the new paradigms and resulting benefits.

In this paper a concept of a mixed-reality based learning environment, combining physical objects and visualisation of its digital counterpart was introduced. It allows making these complex and invisible relations experienceable and thereby understandable for todays and tomorrow's production environments. Thereby, it was designed to not address one specific discipline but help various people from several disciplines to gain a deeper understanding. Furthermore, the expected benefits were outlined and estimated. Ideas how such a learning environment can be included in existing academic and advanced training curricula were presented as well. The relevant State of the Art for reality-based interaction, gamification, and blended learning was described and related trends and requirements listed.

\section{References}

- [1] H. Kagermann, J. Helbig, A. Hellinger, and W. Wahlster, "Recommendations for Implementing the Strategic Initiative INDUSTRIE 4.0: Securing the Future of German Manufacturing Industry; Final Report of the Industrie 4.0 Working Group,” 2013.

- [2] A. Tang, C. Owen, F. Biocca, and W. Mou, "Comparative effectiveness of augmented reality in object assembly," in Proceedings of the SIGCHI conference on Human factors in computing systems, 2003, pp. 73-80.

- [3] K. Mura, D. Gorecky, and G. Meixner, "Involving Users in the Design of Augmented Reality-Based Assistance in Industrial Assembly Tasks," in Applied Human Factors and Ergonomics, 2012.

- [4] D. Gorecky, G. Lawson, K. Mura, S. Hermawati, and M. L. Overby, "User-centered design of a training system," in Applied Human Factors and Ergonomics, 2012.

- [5] N. Petersen and D. Stricker, "Learning task structure from video examples for workflow tracking and authoring,” Mix. Augment. Real. ..., 2012.

- [6] D. Gorecky, R. Campos, and G. Meixner, "Seamless Augmented Reality Support On The Shopfloor Based On Cyber-Physical-Systems,” in Proceedings of MobileCHI 2012, 2012.

- [7] E. Eiriksdottir and R. Catrambone, "Procedural Instructions, Principles, and Examples: How to Structure Instructions for Procedural Tasks to Enhance Performance, Learning, and Transfer,” Hum. Factors J. Hum. Factors Ergon. Soc., vol. 53, no. 6, pp. 749-770, 2011.

- [8] K. Rixecker, "Lego für das Internet der Dinge: MESH soll jeden zum Maker machen," t3n.de, 2015. [Online]. Available: http://t3n.de/news/lego-fuer-internet-dinge-mesh-592906/.

- [9] S. R. Klemmer, B. Hartmann, and L. Takayama, "How bodies matter: five themes for interaction design," in

- DIS '06: Proceedings of the 6th conference on Designing Interactive systems, 2006, pp. 140-149.

- $\quad$ [10] R. Jacob, A. Girouard, L. Hirshfield, M. Horn, O. Shaer, E. Solovey, and J. Zigelbaum, "Reality-based interaction: a framework for post-WIMP interfaces," in CHI, 2008.

- [11] H. C. Jetter, H. Reiterer, and F. Geyer, "Blended Interaction: Understanding natural human-computer interaction in post-WIMP interactive spaces,” Pers. Ubiquitous Comput., vol. 18, pp. 1139-1158, 2014.

- [12] FestoDidactic, “Tec2Screen,” 2015. [Online]. Available: http://www.festo-didactic.com/dede/lernsysteme/. [13] Fraunhofer, “3D-Layoutplanung mit dem IPA-Planungstisch,” 2015. [Online]. Available:

- http://www.ipa.fraunhofer.de/3D-Layoutplanung_mit_dem_IPA-Planungstisch.384.0.html.

- $\quad$ [14] S. Deterding, M. Sicart, L. Nacke, K. O’Hara, and D. Dixon, “Gamification: Using gamedesign elements in non-gaming contexts," in Proceedings of the 2011 annual conference extended abstracts on Human factors in computing systems - CHI EA '11, 2011, p. 2425. 
- [15] O. Korn, "Industrial playgrounds: how gamification helps to enrich work for elderly or impaired persons in production,” Proc. 4th ACM SIGCHI Symp. Eng. Interact. Comput. Syst. EICS '12, p. 313, 2012.

- [16] O. Korn, A. Schmidt, and T. Hörz, "The potentials of in-situ-projection for augmented workplaces in production. A study with impaired persons," in Extended Abstracts of ACM CHI Conference on Human Factors in Computing Systems, 2013, p. 979.

- [17] S. Sonnentag, C. Niessen, and S. Ohly, "Learning at work: Training and development," in International Review of Industrial and Organizational Psychology, Volume 19, C. L. Cooper and I. T. Robertson, Eds. Chichester: John Wiley \& Sons, 2004, pp. 249-289.

- [18] S. Debowski, R. E. Wood, and a Bandura, "Impact of guided exploration and enactive exploration on self- regulatory mechanisms and information acquisition through electronic search.,” J. Appl. Psychol., vol. 86, no. 6, pp. 1129-1141, 2001.

- [19] K. A. Ericsson, "Deliberate practice and the acquisition and maintenance of expert performance in medicine and related domains.,” Acad. Med., vol. 79, no. 10, pp. 70-81, 2004.

- [20] M. Tisch, S. Adolph, J. Metternich, T. Bauernhandl, and G. Reinhart, "Innovative Ansätze zur Kompetenzentwicklung für die Produktion der Zukunft,” Zeitschrift für wirtschaftlichen Fabrikbetr., vol. 109, no. 9, pp. 587-590, 2014.

- [21] B. Heyse and J. Erpenbeck, Kompetenztraining. Informations- und Trainingsprogramme. Stuttgart: Schäffer-Poeschel Verlag, 2009.

- $\quad$ 22] S. Gully and S. Payne, "The impact of error training and individual differences on training outcomes: an attribute-treatment interaction perspective.,” J. Appl. ..., 2002.

- [23] J. Tracey, "Applying trained skills on the job: The importance of the work environment.," J. Appl. ..., 1995.

- [24] M. Schmitt, M. Loskyll, and D. Zühlke, "Development of a Framework for Dynamic Function Deploymentand Extension by Using Apps on Intelligent Field Devices," in The 19th World Congress of the International Federation of Automatic Control. World Congress of the International Federation of Automatic Control (IFAC-14), Promoting automatic control for the benefit of humankind, 2014. 